\title{
LA PRODUCCIÓN DE ARVEJA (PISUM SATIVUM) EN LA VEREDA MONTEADENTRO, PROVINCIA DE PAMPLONA, NORTE DE SANTANDER
}

\author{
Guillermo Peñaranda Cáceres* \\ Deicy Yuley Molina Gelves**
}

* Docente del departamento de Agrarias Facultad de Ciencias Económicas y Empresariales Universidad de Pamplona. Pamplona Colombia. E-mail: german1952@mail.ru

*** Economista de la Universidad de Pamplona. Grupo de investigación GIAS, Facultad de Ciencias Agrarias 


\section{LA PRODUCCIÓN DE ARVEJA (PISUM SATIVUM) EN LA VEREDA MONTEADENTRO, PROVINCIA DE PAMPLONA, NORTE DE SANTANDER}

\begin{abstract}
RESUMEN
La producción de arveja ocupa un lugar muy importante en la vereda Monteadentro, del municipio de Pamplona, ya que gran parte de sus habitantes tiene dedicada sus tierras a la producción de este rubro. El aporte económico de este rubro es primordial para su subsistencia y para el desarrollo económico y agrícola de la provincia de Pamplona, cabe destacar la influencia de producción del cultivo de arveja en la vereda de Monteadentro, que se constituye como alternativa para el desarrollo de la economía local, la generación de empleo e ingresos. La vereda cuenta con condiciones climáticas optimas (entre los 10 y $17^{\circ} \mathrm{C}$ ), de suelo (la mayoría de los suelos de la vereda contienen fosforo o potasio por lo tanto es innecesario el uso de químicos que contengan estos elementos lo que repercute en menores costos de producción), y además de buena disponibilidad de la mano de obra (la mayoría de los habitantes tiene entre 20 y 45 años, aunque no estén capacitados en cuanto al proceso precosecha, solo producción artesanal), que de alguna manera ha influido en el reconocimiento de la producción de dicho cultivo a nivel regional.
\end{abstract}

Palabras Claves: Arveja, costos de producción, productividad, variedades, valor agregado.

\begin{abstract}
Pea production occupies an important place in the village Monteadentro, municipality of Pamplona, and much of its population, has dedicated its land to the production of this item. The economic contribution of this sector is essential for subsistence and for economic and agricultural development in the province of Pamplona. Include the influence of pea crop production in the village of Monteadentro, which is formed as an alternative for local economic development, employment generation and income. The trail has optimal climatic conditions (between 10 and $17^{\circ} \mathrm{C}$ ), soil (most of the soils of the path containing phosphorus or potassium is therefore unnecessary to use chemicals containing these elements that impact on lower costs production), and in addition to good availability of labor (the majority of the population is between 20 and 45, although not trained in pre-harvest process, only small-scale production), which has somehow influenced recognition of that crop production at regional level.
\end{abstract}

Key Words: Pea, production costs, productivity, variety, value added 


\section{ANTECEDENTES HISTÓRICOS}

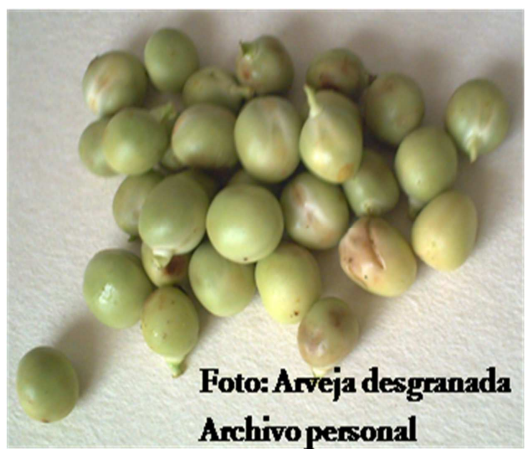

La arveja o guisante (Pisum sativum) se ha cultivado en Europa durante siglos y se cuenta hoy día entre las hortalizas más populares en todo el mundo, y la forma de consumo más popular es enlatada, como frutos secos o congelados (de los distintos tipos de conservación de alimentos, el congelado es uno de los que más se asemeja al estado natural del producto original) ${ }^{1}$. Cuando se consumen en fresco, procedentes del huerto y cocinados de manera adecuada, constituyen una hortaliza especialmente suculenta. La arveja verde es una de las leguminosas que mayor cantidad de carbohidratos y proteínas entrega por unidad de peso, destacándose como fuente importante de sacarosa y aminoácidos, incluyendo lisina. El desarrollo de la planta tiene unas etapas definidas las cuales permiten el control de plagas y enfermedades de manera efectiva, además es un alimento de contenidos significativos de minerales ( $\mathrm{P}$ y $\mathrm{Fe}$ ) y de vitaminas, especialmente B1. ${ }^{2}$

Se han encontrado restos fosilizados de guisantes en yacimientos arqueológicos del Próximo Oriente que datan de hace casi 10.000 años. Las especies cultivares aparecieron relativamente poco después del trigo y la cebada, por lo que se supone que ya se cultivaban hacia el 7.800 a. C. En el 2.001 a. C. su cultivo se había extendido por Europa y hacia el este a la India, aunque hasta el siglo XVI sólo se usaba en grano seco o como forraje. A partir de ese momento, empezó a usarse también el grano fresco. El principal productor de arveja fresca es India con el 65\% del total, y le sigue en importancia Pakistán: Entre ambos países producen el $90 \%$ del total mundial. Muy distante ocupa el tercer puesto Turquía, que cosecha el 5-6\% del volumen mundial, mientras que el mayor productor de arvejas secas (guisantes secos) del año 2007 es Canadá $^{3}$, que produjo el 29,9\% de un volumen mundial de 10.128 .486 toneladas, seguido por China y la Federación Rusa participaron con el 13,8\% y el 8,6\%, respectivamente, ubicándose como el segundo y el tercer productor de arvejas.

\footnotetext{
${ }^{1}$ MACHADO, Absalón C. y TORRES, Jorge. 1991

2 http:/ /www.fenalce.org/pagina.php?p_a $=52$

${ }^{3} \mathrm{FAO}$
} 


\section{PRODUCCIÓN DE LA ARVEJA EN COLOMBIA}

La arveja es considerada como uno de los productos básicos de la economía campesina de pequeños y medianos productores, ubicados especialmente en la zona andina. Este cultivo, sobresaliente en varias regiones del territorio nacional (se produce en 11 de sus 32 departamentos), especialmente en el clima frío y medio, localizados en el altiplano cundíboyacense y en los departamentos de Nariño y Tolima, entre los 2200 y 3000 msnm (ya que posibilita su crecimiento, producción y control de plagas), su producción es muy sencilla y además muy provechosa (tan solo con 1 kilo de semilla sembrada se puede recoger 125 kilos de arveja fresca en vaina) ${ }^{4}$. La semilla es el principal aprovechamiento de este grupo de leguminosas, las cuales son cultivadas como alimento, forraje, en verde o heno, y juegan un papel importante como abono verde, enterrado al final de la cosecha para mejorar la fertilidad de los suelos. Con unas 10 a 15 plantas, se puede obtener la cantidad de granos suficientes para el consumo normal de sabrosas arvejas frescas de una familia de 4 miembros $^{5}$. La adecuada selección de la semilla, la rotación correcta de cultivos, el tutorado (colgada), la buena preparación del suelo, la fertilización adecuada y el oportuno control de malezas, disminuyen la incidencia y severidad de las enfermedades en el cultivo de arveja.

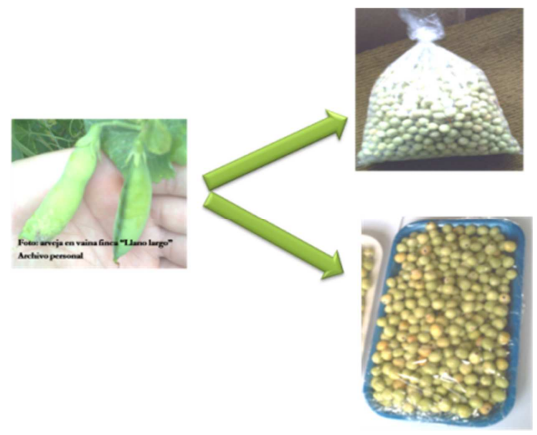

En Colombia la arveja es la leguminosa que ocupa el segundo lugar en orden de importancia después del fríjol, por el área cultivada y por ser fuente de proteína, $(22 \%$ y $25 \%)$, constituyéndose en un alimento básico de la canasta familiar ${ }^{6}$. En el 20057, se registro una producción de 34.310 t, muy por debajo de su potencial, ya que ocupo tan solo 26.795 ha, siendo representativa la siembra de la variedad Santa Isabel, variedad indeterminada, se cosecha entre 115 y 145 días en verde y hasta 160 días en seco $^{8}$, sus rendimientos fluctúan entre 900 y $1.200 \mathrm{~kg} \cdot \mathrm{ha}-1$. Es de anotar que Colombia tiene zonas aptas para su producción, cubrir la demanda interna y exportar. Las importaciones de arveja seca durante el primer semestre del 2009 disminuyeron con respecto a lo visto durante el primer semestre de 2008, pasando de 19,983 a 15,842 toneladas, lo que equivale a un $21 \%$ menos $^{9}$, siendo representativo para el mercado nacional, ya que permite impulsar

\footnotetext{
${ }^{4}$ Asociación de productores agropecuarios, ASPAGRO, análisis de la producción de arveja en el municipio de Pamplona, Norte de Santander.

${ }^{5}$ SERRA, Daniel Oscar. http://usuarios.lycos.es/dserra/huertayjardineria/arvejas.htm. Córdoba, República de Argentina

${ }^{6}$ GONZÁLEZ, 2000

7 Agro net, 2007

8 Sánchez y Mosquera, 2006; Buitrago et al., 2006

${ }^{9}$ Fondo nacional de leguminosas. Tami, Lina y Alkhatib Abdala, Samira. Abril-Junio 2009
} 
la agricultura y fortalecer la producción del cultivo, al respecto opina Robledo ${ }^{10}$, "Impulsar al máximo la agricultura nacional obedece a una concepción democrática y soberana del desarrollo económico". Dentro de las estrategias que se pueden efectuar para el cultivo de arveja, cabe mencionar una transformación productiva, dar una utilidad adicional cambiando los métodos de producción, es decir, tecnificar, especializar y diversificar los cultivos para obtener una mayor competitividad, teniendo en cuenta las ventajas comparativas. Ostertag y Rizo ${ }^{11}$, consideran que un producto con valor agregado es aquel al cual se le hace una o más operaciones con el fin de adecuarlo a los requerimientos de los compradores, sean clientes industriales o consumidores finales.

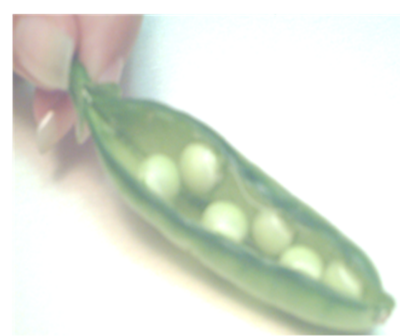

Foto: Arveja en vaina Archivo personal

Para el desarrollo de la economía nacional la siembra del guisante se considera un actor estratégico, ya que tiene un periodo y unos costos de producción relativamente bajos, lo cual permite a la mayoría de los agricultores de clima templado y algo húmedo, donde el desarrollo vegetativo tiene su óptimo de crecimiento con temperaturas comprendidas entre 16 y $20^{\circ} \mathrm{C}$, que se cultive de manera sencilla y sea rentable. Para la provincia de Pamplona, el área sembrada de arveja tecnificada para el $2008^{12}$ fue de 73 hectáreas, con una producción de 584 ton, representando el $46.23 \%$ de la producción total del departamento, se destaca la influencia de producción de arveja (80 bultos semanales) en la vereda de Monteadentro, este se constituye como alternativa para el desarrollo de la economía local, la generación de empleo e ingresos, la zona posee optimas condiciones climáticas (entre los 10 y $17^{\circ} \mathrm{C}$ ), de suelo (la mayoría de los suelos de la vereda contienen buena cantidad de fosforo y potasio, por lo tanto es casi innecesario el uso de agro-químicos que contengan estos elementos lo que repercute en menores costos de producción), y disponibilidad de la mano de obra (la mayoría de los habitantes tiene entre 20 y 45 años), aunque no estén capacitados en cuanto al proceso pre-cosecha, solo producción artesanal), que de alguna manera han provocado el reconocimiento de la producción de dicho cultivo a nivel regional.

\section{PARÁMETROS TÉCNICOS DEL CULTIVO DE ARVEJA}

El cultivo de arveja requiere de condiciones óptimas de humedad del suelo, por lo que necesita poco riego, por tanto en la vereda de Monteadentro, las condiciones del suelo

\footnotetext{
${ }^{10}$ Robledo, Jorge Enrique. Senador. Bogotá.

11 OSTERTAG, Carlos Felipe y RIZO, Juliana Andrea. Introducción al Valor Agregado. Proyecto de Desarrollo Agro-empresarial Rural. Marzo de 2002

12 Anuario estadístico 2008
} 
se caracterizan por ser las mejores para este cultivo, ya que la arveja es poco exigente en abonos minerales, pero aun así es aconsejable aplicar antes de la siembra (realizando un previo análisis de suelos), unos 25 gramos por metro cuadrado de abono complejo 815-15 o de 15-15-15, lo que indica que si es necesario la utilización de abonos químicos para su manutención pero no en excesiva cantidad y algunas veces por los resultados del estudio de suelos que se hace previamente debería ser por poco o nula la aplicación de estos químicos.

\section{Tabla 1 Requerimientos de macro elementos para arveja según la casa Rogers NK}

\begin{tabular}{|c|c|}
\hline ELEMENTO & KILOS / HA \\
\hline Nitrógeno (N) & 63 \\
\hline Fósforo (P2O5) & 95 \\
\hline Potasio (K2O) & 106 \\
\hline \multicolumn{2}{|c|}{ Fuente: Rogers NK } \\
\hline
\end{tabular}

En los cultivos de la vereda de Monteadentro se encontró que actualmente debido a la falta de asistencia técnica especializada los productores se están guiando por formulación anterior (tabla 1). Sabemos que las plantas necesitan alimento el cual toman del suelo; pero después de varios años, los nutrientes se agotan y es preciso reponerlos para continuar cosechando buenos productos en cantidad y calidad, como podemos ver en la tabla 1 los macro elementos que necesita la arveja son nitrógeno, fosforo y potasio, los cuales fortalecen y dan protección a la planta para su crecimiento. Los nutrientes mayores que necesita en mayor cantidad la planta son:

a. Nitrógeno (N): Influye directamente en el crecimiento de la planta; cuando hay mucho Nitrógeno es posible que retarde la maduración y fructificación, forma parte de la clorofila, proteínas y vitaminas, ayuda a la asimilación de otros nutrientes.

b. Fósforo (P). Contribuye a: la buena formación de la semilla; flores y frutos bien desarrollados; raíces fuertes y abundantes y la resistencia a varias enfermedades.

c. Potasio (K). Contribuye a: formación de un buen sistema de raíces; tallos fuertes y vigorosos; azúcares, almidones y proteínas; aumenta el peso de granos y semillas y contribuye a dar resistencia contra las enfermedades.

A partir el análisis efectuado en la vereda se encontró que las principales labores en el cultivo de la arveja son:
a. Trazado
b. Siembra
c. Desyerbe
d. Raleo 

e. Defoliación
f. Cosecha
g. Selección
h. Empaque

Herramientas para ejecutar las labores: Pala, pica, azadón, bueyes, arado.

Control de malezas: puede ser mecánico o químico.

El control mecánico se realiza mediante una deshierba manual cuando las plantas alcanzan 15 centímetros de altura con guadañadora. El control químico se efectúa mediante la aplicación de herbicidas, en el caso de malezas de hoja ancha se esta recomendando la aplicación pos emergente de Sencor (metribuzina) en dosis de 400 gramos por hectárea; esta aplicación la realizan cuando la maleza tenga máximo 3 hojas pequeñas y la planta de arveja tenga entre 15 y 20 centímetros de altura. Cuando las malezas son gramíneas (tres hojas pequeñas) se controla con aplicaciones pos emergentes de Fusilade 2000 (floazifob-butil) en dosis de 2 litros por hectárea.

\section{SISTEMAS DE PRODUCCIÓN}

El sistema de siembra empleado en la zona es la siembra en ladera y en surcos en contra de la pendiente arando con bueyes.. El desarrollo vegetativo de la producción de arveja tiene su óptimo de crecimiento con temperaturas comprendidas entre $16^{\circ} \mathrm{C}$ y $20^{\circ} \mathrm{C}$, estando el mínimo entre $6^{\circ} \mathrm{C}$ y $10^{\circ} \mathrm{C}$ y el máximo en $35^{\circ} \mathrm{C}$.

Época de cosecha Enero - Mayo y debe coincidir con el tiempo seco, para evitar daño en la calidad del producto. Para la cosecha en vaina verde se hacen dos pases, el primero a los 105 días y el segundo dos semanas más tarde.

\section{VARIEDADES}

Las variedades existentes presentan características de acuerdo al suelo y al mejoramiento genético que se ha desarrollado en cada región, por ejemplo podemos mencionar: Piquinegra u Ojinegra ${ }^{13}$, Crema Lisa, Verde Seca, Parda, Pajarito, Guatecaná, Bogotana, Diacol Boyacá, Diacol Caldas, ICA Boyacá, ICA Teusacá, Alaska, Perfection y Trojan.

En el mercado internacional hay más de 56 variedades inscriptas ${ }^{14}$ como comerciales, la gran mayoría de origen Estadounidense (34\%), Holandés (15\%) e Italianas (13\%), todas ellas con características diferenciales que van desde el color de la semilla (verdes y

13 VALDERRAMA, H. Medardo. et al. Manual práctico ilustrado de hortalizas. Bogotá, 2008. p 37

14 Instituto nacional de semillas, Argentina. 
amarillas), textura del grano (lisas y rugosas), característica de la planta (foliosas y semiáfilas o zarcillosas), y con peso de 1000 semillas que van entre los 150 gr hasta más de 300 gr. Se comercializan las siguientes variedades:

- Siglo, Lenca y Trapper, origen canadiense

- Teléfono y Enano, origen norteamericano.

- Negret, origen inglés.

- Voluntario y Televisión, origen francés.

- Miranda y Paloma, origen Países Bajos

- Tirabeque, origen español.

- Arveja proteica, Facón y Viper, origen argentino

- Amarilla-INIA y Finale, origen Chileno

\section{ANÁLISIS DE COSTOS DE PRODUCCIÓN PRE COSECHA}

La tabla 2, nos muestra los costos fijos ${ }^{15}$ del cultivo de arveja en la vereda de Monteadentro, en donde los costos directos representan el $89.4 \%$ del total de la producción, en este se incluyen las labores y los insumos utilizados en el proceso productivo, teniendo mayor peso el costo de recolección, el cual requiere de mayores jornales y de tiempo, esto teniendo en cuenta que durante el proceso pre-cosecha se tuvo una densidad de siembra de 25 kilos de semilla por 3/4 de hectárea, con los que normalmente trabajan los agricultores de la vereda. Se encontró que el tipo de semilla a sembrar es la variedad Piquinegra, con un nivel de tecnología artesanal y una distancia de siembra entre surcos de 1 a 1,20 m y entre plantas de 3 a $5 \mathrm{~cm}$.

Tabla 2: Costos de producción cultivo de arveja Piquinegra en la vereda de Monteadentro por cosecha en 0.75 ha

\begin{tabular}{|c|c|c|l|l|c|}
\hline \multirow{2}{*}{$\begin{array}{c}\text { ACTIVIDADE } \\
\text { S }\end{array}$} & \multicolumn{2}{|c|}{ PATRON } & \multicolumn{3}{c|}{2008} \\
\cline { 2 - 6 } & UNIDAD & CANT & PRECIO/UNI & $\begin{array}{c}\% \\
\text { PARTICIPACIÓN }\end{array}$ & $\begin{array}{c}\text { VALOR } \\
\text { TOTAL }\end{array}$ \\
\hline COSTOS & & & & & \\
DIRECTOS & & & & & \\
\hline LABORES & & & & & \\
\hline
\end{tabular}

${ }^{15}$ Estos costos no incluyen el jornal arado (el dueño del cultivo realiza esta labor solo o en compañía de sus hijos), arrendamiento (muchas veces el terreno es propio), estacas, alambre liso y de púas, fumigadora, azadones surtidores, manguera $3 / 4 \mathrm{y}$ arado, estos son elementos utilizados durante el proceso pre cosecha, son permanentes ya que su costo no se incluye al momento de reiniciar la cosecha en el siguiente periodo productivo. 


\begin{tabular}{|c|c|c|c|c|c|}
\hline Rastrillada & jornal & 5,0 & $\$ 20.000$ & $1,9 \%$ & $\$ 100.000$ \\
\hline $\begin{array}{c}\text { Siembre y } \\
\text { tapada - } \\
\text { abonada } \\
\end{array}$ & jornal & 18,0 & $\$ 20.000$ & $7,0 \%$ & $\$ 360.000$ \\
\hline $\begin{array}{l}\text { Envarada - } \\
\text { alambrada }\end{array}$ & jornal & 10,0 & $\$ 20.000$ & $3,9 \%$ & $\$ 200.000$ \\
\hline Aporques & jornal & 10,0 & $\$ 20.000$ & $3,9 \%$ & $\$ 200.000$ \\
\hline $\begin{array}{l}\text { Amarres - } \\
\text { colgada - } \\
\text { tutorado }\end{array}$ & jornal & 15,0 & $\$ 20.000$ & $5,8 \%$ & $\$ 300.000$ \\
\hline $\begin{array}{l}\text { Aplicación de } \\
\text { fungicidas }\end{array}$ & jornal & 15,0 & $\$ 20.000$ & $5,8 \%$ & $\$ 300.000$ \\
\hline $\begin{array}{l}\text { Aplicación de } \\
\text { insecticidas }\end{array}$ & jornal & 4,0 & $\$ 20.000$ & $1,5 \%$ & $\$ 80.000$ \\
\hline $\begin{array}{l}\text { Aplicación de } \\
\text { herbicidas }\end{array}$ & jornal & 2,0 & $\$ 20.000$ & $0,8 \%$ & $\$ 40.000$ \\
\hline Recolección & jornal & 40,0 & $\$ 20.000$ & $15,5 \%$ & $\$ 800.000$ \\
\hline Alquiler bueyes & animales & 2,0 & $\$ 40.000$ & $1,5 \%$ & $\$ 80.000$ \\
\hline Transporte & global & 1,0 & $\$ 80.000$ & $1,5 \%$ & $\$ 80.000$ \\
\hline SUBTOTAL & & & & $49,1 \%$ & $\begin{array}{c}\$ \\
2.540 .000 \\
\end{array}$ \\
\hline \multicolumn{6}{|l|}{ INSUMOS } \\
\hline Semillas & $\mathrm{Kg}$ & 25 & $\$ 8.000$ & $3,9 \%$ & $\$ 200.000$ \\
\hline $\begin{array}{c}\text { Compuestos } \\
\text { (Abonos) }\end{array}$ & bultos & 6 & $\$ 100.000$ & $11,6 \%$ & $\$ 600.000$ \\
\hline Foliares & Lt & 3 & $\$ 20.000$ & $1,2 \%$ & $\$ 60.000$ \\
\hline $\begin{array}{l}\text { Herbicida } \\
\text { (Falon) }\end{array}$ & $250 \mathrm{gr}$ & 2 & $\$ 32.000$ & $1,2 \%$ & $\$ 64.000$ \\
\hline $\begin{array}{c}\text { Insecticida } \\
\text { (Sistemin) }\end{array}$ & $500 \mathrm{~cm}$ & 2 & $\$ 30.000$ & $1,2 \%$ & $\$ 60.000$ \\
\hline $\begin{array}{c}\text { Fungicida } \\
\text { (Fictoras y/o } \\
\text { Tribian) }\end{array}$ & $500 \mathrm{gr}$ & 15 & $\$ 17.500$ & $5,1 \%$ & $\$ 262.500$ \\
\hline $\begin{array}{c}\text { Fungicida } \\
\text { (Folicur) }\end{array}$ & $250 \mathrm{~cm}$ & 6 & $\$ 40.000$ & $4,6 \%$ & $\$ 240.000$ \\
\hline Abono orgánico & bultos & 12 & $\$ 15.000$ & $3,5 \%$ & $\$ 180.000$ \\
\hline Empaques & pares & 40 & $\$ 2.000$ & $1,5 \%$ & $\$ 80.000$ \\
\hline $\begin{array}{c}\text { Cabuya - } \\
\text { Hilazas }\end{array}$ & Cono & 30 & $\$ 9.000$ & $5,2 \%$ & $\$ 270.000$ \\
\hline cal & bultos & 10 & $\$ 7.000$ & $1,4 \%$ & $\$ 70.000$ \\
\hline SUBTOTAL & & & & $40.3 \%$ & $\$$ \\
\hline
\end{tabular}




\begin{tabular}{|c|c|c|c|c|c|} 
& & & & 2.086 .500 \\
\hline $\begin{array}{c}\text { TOT. COSTOS } \\
\text { DIRECTOS }\end{array}$ & & & & $89,4 \%$ & 4.626 .500 \\
\hline $\begin{array}{c}\text { COSTOS } \\
\text { INDIRECTOS }\end{array}$ & & & & & \\
\hline Arrendamiento & mes & 5,0 & $\$ 100.000$ & $9,7 \%$ & $\$ 500.000$ \\
\hline Imprevistos & semestre & & $\$ 50.000$ & $1,0 \%$ & $\$ 50.000$ \\
\hline $\begin{array}{c}\text { TOT COST. } \\
\text { INDIRECTOS. }\end{array}$ & & & & $10,6 \%$ & $\$ 550.000$ \\
\hline $\begin{array}{c}\text { PROMEDIO.P } \\
\text { RODUCCION }\end{array}$ & Bultos & 80 & $\$ 63.831$ & $100,0 \%$ & $\$ 5.176 .500$ \\
\hline
\end{tabular}

Gráfica 1. Incidencia porcentual de grandes rubros en los costos de producción de arveja

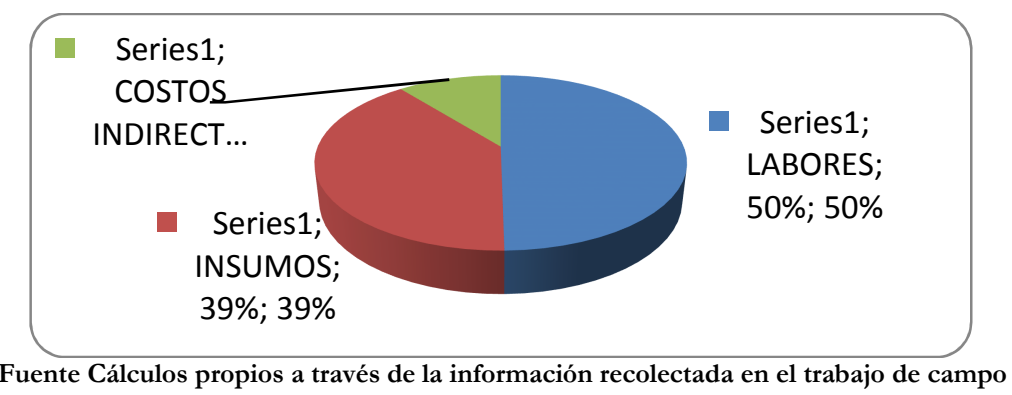

El análisis a estos datos señalan que la mano de obra tiene una gran incidencia en los costos de producción ocupando el 50 por ciento de estos, lo que nos indica que el cultivo es artesanal en el laboreo de las tareas que se efectúan en el cultivo (el único implemento mecánico es la guadañadora), y que persiste el uso de agroquímicos, ocupando el 39 por ciento de los costos. Cabe señalar que aun no se emplean plantas alelopáticas o productos naturistas de tecnologías alternativas, que podrían reducir los costos de producción en este rubro.

\section{COSTOS POS-COSECHA CULTIVO DE ARVEJA}

Teniendo en cuenta los costos pos cosecha para un bulto (50 kilos) de arveja fresca en vaina, se analizara el proceso de desgranado y congelado, se analizo el proceso de desgranado y congelado, según los cálculos realizados, el bulto tiene un rendimiento del 85\% (42.5 kilos) desgranado, se ofrecen en presentaciones de libra (21.5 kilos) y de kilo (21 kilos) para satisfacer las necesidades del mercado. Una transformación productiva para consumir el producto en congelado se relaciona con la comodidad, facilidad y 
rapidez en la preparación de una porción, con los mismos nutrientes que los productos en fresco. Cuando la producción es alta y los precios disminuyen, se puede realizar el siguiente procedimiento denominado "blanqueado": Someter los granos a un rápido hervor, 5 minutos y luego enfriarlos inmediatamente con agua helada, escurrirlos, colocarlos en una bolsa de polietileno para freezer y frizarlos inmediatamente. Conservan todas sus características originales de arvejas frescas por mucho tiempo. ${ }^{16}$

Tabla 3: Costos de transformación de la arveja (pos cosecha)

Fuente: Cálculos propios

\begin{tabular}{|c|c|c|c|c|c|}
\hline & \multicolumn{2}{|c|}{ PATRON } & \multicolumn{3}{|c|}{2008} \\
\hline ACTIVIDADES & UNIDAD & CANT. & $\begin{array}{l}\text { PRECIO/ } \\
\text { UNIDAD }\end{array}$ & $\%$ PART. & $\begin{array}{l}\text { VALOR } \\
\text { TOTAL }\end{array}$ \\
\hline peso tipo reloj* & unidad & 1 & $\$ 40.000$ & $32,4 \%$ & $\$ 40.000$ \\
\hline \multicolumn{6}{|l|}{$\begin{array}{c}\text { ARVEJA } \\
\text { DESGRANADA }\end{array}$} \\
\hline $\begin{array}{l}\text { bolsa plástica } \\
\text { transparente } 1 \mathrm{~b}\end{array}$ & unidad & 43 & $\$ 10$ & $0,3 \%$ & $\$ 430$ \\
\hline $\begin{array}{l}\text { bolsa plástica } \\
\text { transparente } \mathrm{kg}\end{array}$ & unidad & 21 & $\$ 15$ & $0,3 \%$ & $\$ 315$ \\
\hline \multicolumn{6}{|l|}{$\begin{array}{c}\text { ARVEJA } \\
\text { CONGELADA }\end{array}$} \\
\hline $\begin{array}{c}\text { bandeja de icopor } \\
\text { pequeña }\end{array}$ & unidad & 43 & $\$ 30$ & $1,0 \%$ & $\$ 1.290$ \\
\hline $\begin{array}{c}\text { bandeja de icopor } \\
\text { grande }\end{array}$ & unidad & 21 & $\$ 40$ & $0,7 \%$ & $\$ 840$ \\
\hline papel transparente & Rollo (100 ms) & 1 & $\$ 20.000$ & $16,2 \%$ & $\$ 20.000$ \\
\hline olla de 25 litros cc $*$ & unidad & 1 & $\$ 40.000$ & $32,4 \%$ & $\$ 40.000$ \\
\hline $\begin{array}{c}\text { gas propano } 33 \\
\text { libras } * 17\end{array}$ & cocción & 1 & $\$ 20.400$ & $16,5 \%$ & $\$ 20.400$ \\
\hline \multicolumn{4}{|c|}{ TOTAL COSTOS POSCOSECHA } & $100,0 \%$ & $\$ 123.275$ \\
\hline
\end{tabular}

\footnotetext{
${ }^{16}$ www.airesdehuerta.com.ar/arveja/

*Elementos utilizados durante el proceso pos cosecha, son permanentes o se utilizan por varios periodos, ya que su costo no se incluye al momento de realizar la siguiente transformación.

*Elementos utilizados durante el proceso pos cosecha, son permanentes o se utilizan por varios periodos, ya que su costo no se incluye al momento de realizar la siguiente transformación.
} 
Teniendo en cuenta los costos pos cosecha para el cultivo de arveja (tabla 3), la importancia de realizar un proceso de transformación productiva radica en la disponibilidad de la materia prima, la producción por familia (3/4 de hectárea) es de 80 bultos semanales de 50 kilogramos, por ello si se logra consolidar, capacitar e incentivar a toda la población para que a través de la transformación productiva de la arveja teniendo en cuenta las diferentes presentaciones: desgranada y/o congelada, se genere valor agregado y en el largo plazo conformar una cadena productiva ayudando al fortalecimiento de las potencialidades tanto de la vereda con respecto al cultivo de arveja, como del valor agregado que se genere, implementando las normas y/o procedimientos necesarios para que el cultivo sea rentable a un menor costo.

\section{Grafica 2 Producción en temporada alta}

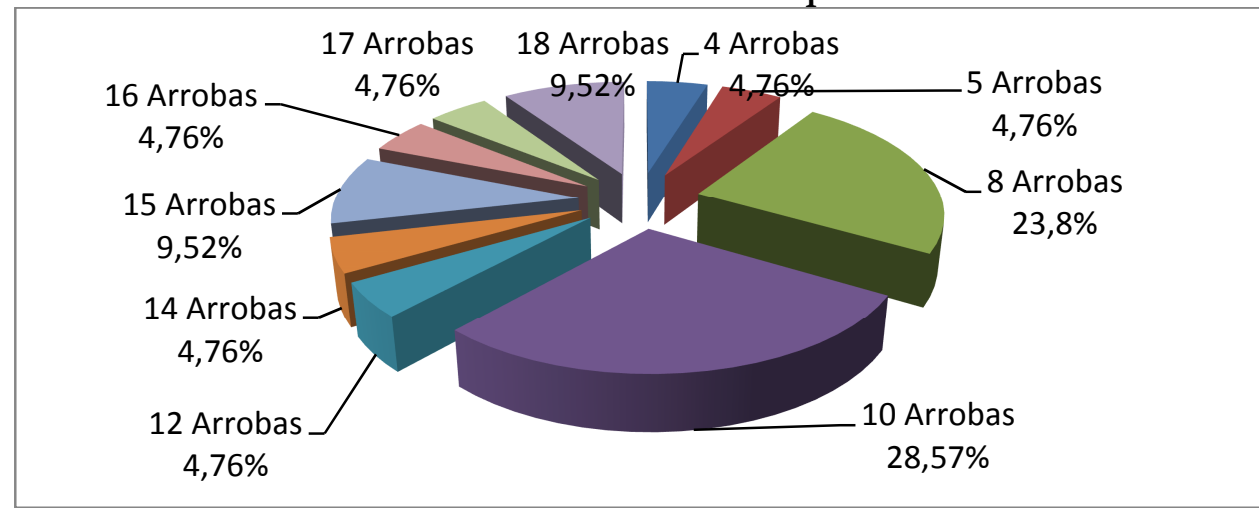

Fuente: Análisis económico de la producción de arveja en la vereda Monteadentro del municipio de Pamplona 2010

Como consecuencia del clima y los suelos, la producción en la vereda de Monteadentro se mantiene constante durante los dos semestres del año, y oscila entre 4 y 20 arrobas, registrándose una participación del $28.57 \%$ en la obtención de 10 arrobas por familia del total de producción de la vereda.

\section{CONCLUSIONES}

Dentro del esquema de desarrollo endógeno podemos mencionar la falta de capacitación y tecnificación de los agricultores para agregar variedades mejoradas a través de la investigación para que los suelos de la vereda de Monteadentro sean mejor y mayor explotados y así obtener una mayor rentabilidad del cultivo.

La producción departamental es significativa en cuanto a hortalizas se refiere, además se pudo identificar que la mayor producción de arveja se encuentra en el municipio de 
Pamplona además de tomate y cebolla, cabe destacar que la producción de arveja es tecnificada, la semilla está calificada para ser sembrada evitando de esta manera el riesgo de enfermedades, que puedan afectar al cultivo en general.

El estudio determino que los productores son poseedores de la tierra que laboran, que en su mayoría son pequeños propietarios, con un bajo nivel de educación. A la problemática regional hay que agregar que no hay asistencia técnica especializada y que no hay ningún organismo estatal que les capacite en organización y planificación de los procesos productivos y en pos cosecha. Del mismo modo en el aspecto de comercialización del producto final, ya que lo hacen en costales, sin desgranar, sin cadena de frio, lo que le podría dar un valor agregado a la producción.

\section{RECOMENDACIONES}

Darle valor agregado a la arveja significa mayor productividad e ingresos para los agricultores de la vereda, por ello es necesaria una política de seguridad alimentaria más efectiva para el agricultor y que permita un desarrollo agro-económico regional más fuerte.

Otra forma de aumentar los ingresos es reducir los costos de producción aplicando una organización más efectiva del trabajo en las labores de siembra y cosecha. Así mismo utilizando productos naturistas para el control de plagas y enfermedades y plantas alelopáticas que alejen insectos e inhiban el crecimiento de malezas. Efectuando esta práctica gastaríamos menos insumos agroquímicos.

Conformar específicamente una asociación de productores de arveja, que tenga como objetivo lograr una mejor calidad del producto, dándole mayor valor agregado a la arveja con utilización de cadena de frio y que comercialice la producción en distintas presentaciones, buscando un mejor mercado.

\section{BIBLIOGRAFÍA}

- AGRONET, 2007. Producción nacional por producto: Arveja. En: http://www.agronet.gov.co/.

- Asociación de productores agropecuarios, ASPAGRO, análisis de la producción de arveja en el municipio de Pamplona, Norte de Santander.

- Federación Nacional de cultivadores de cereales y leguminosas. http://www.fenalce.org/pagina.php?p_a $=52$ 
- FAO, Codex Alimentarius. Proposed draft codex general standard for fruits juices and nectars. Roma

- GONZÁLEZ Arcila, Belén. Proyecto Ajuste, Validación y Transferencia de Tecnología de la Línea OBO-AR-016 Como Nueva Variedad Mejorada de Arveja para la Zona de Economía Campesina del Departamento de Nariño. 2000

- MACHADO, Absalón y TORRES, Jorge. "El sistema agroalimentario: una visión integral de la cuestión agraria en América Latina” 1991

- OStertag, Carlos Felipe y RIZO, Juliana Andrea. Introducción al Valor Agregado. Proyecto de Desarrollo Agro-empresarial Rural. Marzo de 2002

- $\quad$ VALDERRAMA, H. Medardo. Manual práctico ilustrado de hortalizas. Bogotá, 2008. p 37

- ROBLEDO, Jorge Enrique. Libre comercio e importaciones masivas atentan contra la seguridad alimentaria o soberanía alimentaria de Colombia.

- SANCHEZ, E.A. y T. MOSQUERA. 2006. Establecimiento de una metodología para la inducción de re generantes de arveja (Pisum sativum) variedad Santa Isabel. Agronomía Colombiana. 24(1), 17-27.

- SERRA, Daniel Oscar. http://usuarios.lycos.es/dserra/huertayjardineria/arvejas.htm. Córdoba, República de Argentina

- $\quad$ TAMI, Lina y ALKHATIB Abdala, Samira. Abril-Junio 2009. Fondo nacional de leguminosas. Coyuntura cerealista $\mathrm{N}^{\mathrm{o}} 21$ 